\title{
REVIEW: AUDIO NOISE REDUCTION USING FILTERS AND DISCRETE WAVELET TRANSFORMATION
}

\author{
Shalu Rani ${ }^{1 *}$, Rajbhupider Kaur ${ }^{2}$ \\ ${ }^{1}$ Research scholar, ${ }^{2}$ Assistant Professor \\ *1,2 CE Deptt YCOE, Punjabi University, Patiala, India \\ *1Email: sashalluarora@gmail.com
}

*Corresponding Author: -

Email: sashalluarora@gmail.com

\begin{abstract}
: -
In "Audio noise reduction using filters and Discrete Wavelet Transformation" our applications include noise propagation problem in industrial air handling systems, noise in aircrafts and tonal noise from electric power, as well as isolation of vibration from which noise is one kind of sound that is unexpected or undesired. The noise related problem can be divided into non-additive noise and additive noise. The non-additive noise includes multiplier noise and convolution noise, which can be transformed into additive noise through homomorphism transform. The additive noise includes periodical noise, pulse noise, and broadband noise related problems. There are many kinds of broadband noise, which may include heat noise, wind noise, quantization noise, and all kinds of random noise such as white noise and pink noise. In acoustics applications, noise from the surrounding environment severely reduces the quality of speech and audio signals. Therefore, basic linear are used to denoise the audio signals and enhance speech and audio signal quality. Our main objective is to reduce noise from system which is heavily dependent on the specific context and application. As, we want to increase the intelligibility or improve the overall speech perception quality. Such as SNR, PSNR, MSE and the Time to reduce the noise for noisy signals for removing noise.
\end{abstract}

Keywords: Chevshevby Type-1 Filter, butterworth filter, elliptic filter, MSE, SNR, PSNR

\section{(c) $(\$)$}




\section{INTRODUCTION}

Filters are the manipulation of the amplitude and/or phase response of a signal according to their frequency. These are the basic components of all signal processing and -telecommunication systems. There are two kinds of filters- fixed and tunable. Fixed filters are those in which passband frequencies and stopband frequencies are fixed whereas in case of tunable filters, passband and stopband frequencies are variable. These frequencies can be changed according to the requirement of the applications. Tunable digital filters are widely employed in telecommunications, medical electronics, digital audio equipment and control systems. These filters are also known as variable digital filters [1]. Tunable digital filters are used in telecommunication system in the front end of a receiver to select a particular band of frequencies. In medical electronics, tunable notch filters are used to

Suppress the power line interference [1]. The bases for the design of the tunable digital filters are the spectral transformation. It is basically used to modify characteristics of a filter to meet new specifications without repeating the filter design procedure. This modification is done by changing a Low pass (LP) digital

Filters to Low pass (LP) filters with different cut-off frequencies or to a High pass (HP), Band pass (BP) or Band stop(BS) filters. The variable Band pass (BP) and Band stop (BS) filters are used to eliminate and retrieve some narrow band signals. In [3] variable band pass and band stop filters are shown with high accuracy and independent tuning characteristics.

\section{Audio Noise Reduction}

Audio noise reduction system is the system that is used to remove the noise from the audio signals. Audio noise reduction systems can be divided into two basic approaches. The first approach is the complementary type which involves compressing the audio signal in some well-defined manner before it is recorded (primarily on tape). The second approach is the single- ended or non-complementary type which utilizes techniques to reduce the noise level already present in the source material - in essence a playback only noise reduction system [4]. This approach is used by the LM1894 integrated circuit, designed specifically for the reduction of audible noise in virtually any audio source. Noise reduction is the process of removing noise from a signal. All recording devices, both analogue or digital, have traits which make them susceptible to noise. Noise can be random or white noise with no coherence, or coherent noise introduced by the device's mechanism or processing algorithms. There is Active noise control (ANC), also known as noise cancellation, or active noise reduction (ANR), is a method for reducing unwanted and unprocessed sound by the addition of a second sound specifically designed to cancel the first[2]. Sound is a pressure wave or we can say sound is the analog signals that are processed according to their frequency, which consists of a compression phase and a rarefaction phase. A noise-cancellation speaker emits a sound wave with the same amplitude but with inverted phase (also known as anti-phase) to the original sound. The waves combine to form a new wave, in a process called interference, and effectively cancel each other out - an effect which is called phase cancellation.

Modern active noise control is generally achieved through the use of analog circuits or digital signal processing. Adaptive algorithms are designed to analyze the waveform of the background no neural noise, then based on the specific algorithm generate a signal that will either phase shift or invert the polarity of the original signal. This anti phase is then amplified and a transducer creates a sound wave directly proportional to the amplitude of the original waveform, creating destructive interference [3]. This effectively reduces the volume of the perceivable noise. The transducer emitting the noise cancellation signal may be located at the location where sound attenuation is wanted (e.g. the user's ear/any music/headphone sound). This requires a much lower power level for cancellation but is effective only for a single user.

\section{Types of Noises}

There are many types and sources of noise or distortions and they include:

1. Electronic noise such as thermal noise and shot noise,

2. Acoustic noise emanating from moving, vibrating or colliding sources such as revolving Machines, moving vehicles, keyboard clicks, wind and rain,

3. Electromagnetic noise that can interfere with the transmission and reception of voice, image and data over the radiofrequency spectrum,

4. Electrostatic noise generated by the presence of a voltage,

5. Communication channel distortion and fading and

6. Quantization noise and lost data packets due to network congestion.

Signal distortion is the term often used to describe a systematic undesirable change in a signal and refers to changes in a signal from the non-ideal characteristics of the communication channel, signal fading reverberations, echo, and multipath reflections and missing samples [10]. Depending on its frequency, spectrum or time characteristics, a noise process is further classified into several categories:

1. White noise: purely random noise has an impulse autocorrelation function and a flat power spectrum.

White noise theoretically contains all frequencies in equal power.

2. Band-limited white noise: Similar to white noise, this is a noise with a flat power spectrum and a limited bandwidth that usually covers the limited spectrum of the device or the signal of interest. The autocorrelation of this noise is sincshaped.

3. Narrowband noise: It is a noise process with a narrow bandwidth such as $50 / 60 \mathrm{~Hz}$ from the electricity supply.

4. Coloured noise: It is non-white noise or any wideband noise whose spectrum has a non-flat shape. Examples are pink noise, brown noise and autoregressive noise. 
5. Impulsive noise: Consists of short-duration pulses of random amplitude, time of occurrence and duration.

6. Transient noise pulses: Consist of relatively long duration noise pulses such as clicks, burst noise etc.

\section{Filter}

Filters are networks that process signals in a frequency- dependent manner. The basic concept of a filter can be explained by examining the frequency dependent nature of the impedance of capacitors and inductors [17]. Filters have many practical applications. A simple, single-pole, low-pass filter (the integrator) is often used to stabilize amplifiers by rolling off the gain at higher frequencies where excessive phase shift may cause oscillations. A simple, single-pole, high- pass filter can be used to block dc offset in high gain amplifiers or single supply circuits [8]. Filters can be used to separate signals, passing those of interest, and attenuating the unwanted frequencies. There are a large number of texts dedicated to filter theory. An ideal filter will have an amplitude response that is unity (or at a fixed gain) for the frequencies of interest (called the pass band) and zero everywhere else (called the stop band). The frequency at which the response changes from passband to stopband is referred to as the cut-off frequency [19].

\section{Basic Linear Design}

The functional complement to the low-pass filter is the high-pass filter. Here, the low frequencies are in the stop-band, and the high frequencies are in the pass band. Figure shows the idealized high-pas

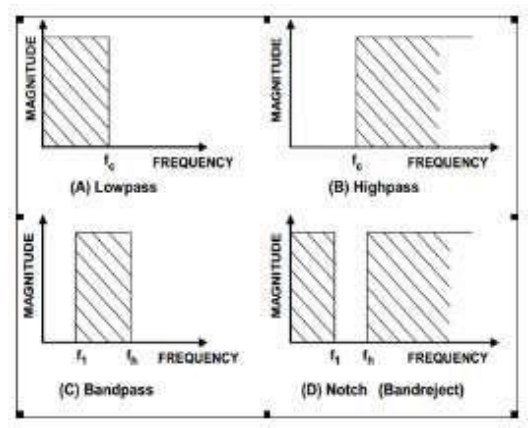

Figure 1.1: Types of Filters [1]

If a high-pass filter and a low-pass filter are cascaded, a band pass filter is created. The band pass filter passes a band of frequencies between a lower cut-off frequency, $\mathrm{f} l$, and an upper cut-off frequency, $\mathrm{f} h$. Frequencies below $\mathrm{f} l$ and above $\mathrm{f}$ $\mathrm{h}$ are in the stop band. An idealized band pass filter is shown in Figure 1.1 [16]. A complement to the band pass filter is the band- reject, or notch filter. The idealized filters defined above, unfortunately, cannot be easily built. The transition from pass band to stop band will not be instantaneous, but instead there will be a transition region. Stop band attenuation will not be infinite [8]

\section{RELATED WORK}

Previous Research work on "Audio noise reduction using filters and Discrete Wavelet Transformation" in the literature survey starting from 2010 to November 2013 was studied. I studied so many papers.

C Mohan Rao et.al [2013] have presents a new adaptive filter whose coefficients are dynamically changing with an evolutionary computation algorithm and hence reducing the noise. This algorithm gives a relationship between the update rate and the minimum error which automatically adjusts the update rate. When the environment is varying, the rate is increased while it would be decreased when the environment is stable and the computation complexity of adaptive filter can be significantly reduced. In the simulation, additive white Gaussian noise is added to the randomly generated information signal and efficiently reduced this noise with minimum or no error by using evolutionary computation with Least Mean Square (LMS) algorithms. Adaptive Noise Cancellation is an alternative way of cancelling noise present in a corrupted signal [1].

K.P. Obulesul et.al [2013] have studied the audio signals are synthetic signals, in which music or speech, are often corrupted by noise during recording and transmission. Speech enhancement is a long-standing problem with numerous applications ranging from hearing aids, to coding and automatic recognition of speech signals etc. and assume that the noise is additive and statistically independent of the signal. Audio denoising procedures are designed to attenuate the noise and retain the signal of interest. Reduction of noise from audio signals has two methods, Diagonal \& Non Diagonal audio denoising algorithms. In this paper, Non diagonal method is used in which Block parameters are automatically adjusted to the nature of the audio signal by minimizing a Stein estimator which is calculated analytically from noisy signal values. This Block thresholding method eliminates "musical noise" by grouping Time-frequency coefficients in blocks before being attenuated [2].

Matheel E. et.al [2009] have presented the digital videos are often corrupted by a noise during the acquisition process, storage and transmission. It made the video in ugly appearance and also affect on another digital video processes like compression, feature extraction and pattern recognition so video denoising is highly desirable process in order to improve the video quality. There are many transformation for denoising process, one of them are Fast Discrete Wavelet 
Transform(FDWT) and framelet transform (Double- Density Wavelet Transform) which is a perfect in denoising process by avoiding the problems in the other transformations. In this paper we propose a method named Translation Invariant with Wiener filter (TIW) this method is proposed to solve the shift variance problem and use this method to denoised a noisy video with Gaussian white noise type. It is applied with Two Dimensional Fast Discrete Wavelet Transform (2-D FDWT), Three Dimensional Fast Discrete Wavelet Transform (3-D FDWT), Two Dimensional Double Density Wavelet Transform (2-D DDWT) and Three Dimensional Double Density Wavelet Transform (3-D DDWT)[3].

Raghavendra Sharma et.al [2008] In this author studied a robust DWPT based adaptive bock algorithm with modified threshold for denoising the sounds of musical instruments shehnai, dafli and flute is proposed. The signal is first segmented into multiple blocks depending upon the minimum mean square criteria in each block, and then thresholding methods are used for each block. All the blocks obtained after denoising the individual block are concatenated to get the final denoised signal. The discrete wavelet packet transform provides more coefficients than the conventional discrete wavelet transform (DWT), representing additional subtle detail of the signal but decision of optimal decomposition level is very important. When the sound signal corrupted with additive white Gaussian noise is passed through this algorithm, the obtained peak signal to noise ratio (PSNR) depends upon the level of decomposition along with shape of the wavelet. Hence, the optimal wavelet and level of decomposition may be different for each signal. The obtained denoised signal with this algorithm is close to the original signal [4].

\section{III.Why to study this?}

The problem undertaken for the dissertation is "Audio noise reduction using filters and Discrete Wavelet Transformation". The Current applications include noise propagation problem in industrial air handling systems, noise in aircrafts and tonal noise from electric power, as well as isolation of vibration from noise is one kind of sound that is unexpected or undesired. The noise related problem that I have studied can be divided into non-additive noise and additive noise. The non- additive noise includes multiplier noise and convolution noise, which can be transformed into additive noise through homomorphism transform. The additive noise includes periodical noise, pulse noise, and broadband noise related problems. The noise generated by the engine is one kind of periodical noise while the one generated from explosion, bump, or discharge is pulse noise problem that I have studied in literature survey. There are many kinds of broadband noise, which may include heat noise, wind noise, quantization noise, and all kinds of random noise such as white noise and pink noise. Therefore, basic linear filters are used to denoise the audio signals and enhance speech and audio signal quality.

\section{Comparison table:}

\begin{tabular}{|c|c|c|c|}
\hline $\begin{array}{l}\text { Author } \\
\text { Name }\end{array}$ & $\begin{array}{l}\text { Year of } \\
\text { Publication }\end{array}$ & $\begin{array}{l}\text { Technique } \\
\text { Used }\end{array}$ & Results \\
\hline $\begin{array}{l}\text { C Mohan } \\
\text { Rao }\end{array}$ & 2013 & $\begin{array}{l}\text { Least } \\
\text { Mean } \\
\text { Square } \\
\text { (LMS) } \\
\text { algorithms }\end{array}$ & $\begin{array}{l}\text { Denoise } \\
\text { the signal } \\
\text { with the } \\
\text { help of } \\
\text { adaptive } \\
\text { signal. }\end{array}$ \\
\hline $\begin{array}{l}\text { K.P. } \\
\text { Obulesul }\end{array}$ & 2013 & $\begin{array}{l}\text { Diagonal } \\
\& \quad \text { Non } \\
\text { Diagonal } \\
\text { audio } \\
\text { denoising } \\
\text { algorithms }\end{array}$ & $\begin{array}{l}\text { Denoise } \\
\text { the signal } \\
\text { with the } \\
\text { help of } \\
\text { Non } \\
\text { Diagonal } \\
\text { audio } \\
\text { algorithm }\end{array}$ \\
\hline $\begin{array}{l}\text { Matheel } \\
\text { E. }\end{array}$ & 2009 & $\begin{array}{l}\text { Fast } \\
\text { Discrete } \\
\text { Wavelet } \\
\text { Transform }\end{array}$ & $\begin{array}{l}\text { Denoise } \\
\text { the signal } \\
\text { with the } \\
\text { help of 2- } \\
\text { D DDWT }\end{array}$ \\
\hline \multirow[t]{2}{*}{$\begin{array}{l}\text { Raghave } \\
\text { ndra } \\
\text { Sharma }\end{array}$} & 2008 & $\begin{array}{l}\text { convention } \\
\text { al discrete } \\
\text { wavelet } \\
\text { transform } \\
\text { (DWT), }\end{array}$ & $\begin{array}{l}\text { To find } \\
\text { the PSNR } \\
\text { of the } \\
\text { denoised } \\
\text { signal }\end{array}$ \\
\hline & & $\begin{array}{l}\text { representin } \\
\mathrm{g} \\
\text { additional } \\
\text { subtle } \\
\text { detail of } \\
\text { the signal } \\
\text { but } \\
\text { decision }\end{array}$ & \\
\hline
\end{tabular}




\section{IV.Conclusion}

In this paper, we have studied filters and discrete wavelet transformation (DWT). Noise problem is major problem. We can apply either filters or DWT. Filters and DWT is combined to remove the noise. Our main motive is to remove the audio noise by applying filters.

\section{References}

[1]. Rao C Mohan et.al “A Variation of LMS Algorithm for Noise Cancellation” International Journal of Advanced Research in Computer and Communication Engineering Vol. 2, ISSN (Print) : 2319-5940, Issue 7, July 2013.

[2]. Obulesu k.p et.al "implementation of time frequency block thresholding algorithm in audio noise reduction "ISSN: 2278 - 7798 International Journal of Science, Engineering and Technology Research (IJSETR) Volume 2, Issue 7, July 2013.

[3]. Abdulmunim Matheel E. et.al "Novel Video Denoising Using 3-D Transformation Techniques "International Journal of Engineering and Advanced Technology (IJEAT) ISSN: 2249 - 8958, Volume-2, Issue-5, June 2013.

[4]. Sharma Raghavendra et.al" A Robust Denoising Algorithm for Sounds of Musical Instruments Using Wavelet Packet Transform "Circuits and Systems, 2013, 4, 459-465 Published Online November 2013.

[5]. Aggarwal Rajeev et.al” Noise Reduction of Speech Signal using Wavelet Transform with Modified Universal Threshold "International Journal of Computer Applications (0975 - 8887) Volume 20- No.5, April 2011.

[6]. Anju et.al "Design of Butterworth and Chebyshev1 Lowpass Filter for Equalized Group Delay" International Journal of Advanced Research in Computer Science and Software Engineering, Volume 2, Issue 5, ISSN: 2277 128X, May 2012.

[7]. Chakraborty Subhadeep et.al" Design of IIR Digital Highpass Butterworth Filter using Analog to Digital Mapping Technique" International Journal of Computer Applications (0975 - 8887) Volume 52 - No. 7, August 2012.

[8]. CHANG S et. al "Speech enhancement for non-stationary noise environment by adaptive wavelet packet". Proceedings of IEEE International Conference on Acoustics Speech and Signal Processing,61-564, 2002.

[9]. Direkoglu C. et.al "Image based multiscale shape description using Gaussian filter", IEEE Indian Conference on Computer Vision, Graphics and Image Processing 673-678, May 2008.

[10]. Direkoglu C et.al "Shape classification using multiscale Fourier-based description in 2-D space", IEEE International Conference on Signal Processing 820-823, June 2008. 